\title{
An Existence Proof for the Hartree-Fock Time-dependent Problem with Bounded Two-Body Interaction
}

\author{
A. Bove \\ Istituto di Fisica dell'Università di Bologna, Bologna, Italy \\ G. Da Prato \\ Istituto Matematico “G. Castelnuovo" dell’Università di Roma, Roma, Italy \\ G. Fano \\ Istituto di Fisica dell’Università di Bologna, Bologna, Italy \\ Received September 19, 1973; in revised form January 4, 1974
}

\begin{abstract}
Using fixed point theorems for local contractions in Banach spaces, an existence and uniqueness proof for the Hartree-Fock time-dependent problem is given in the case of a finite Fermi system interacting via a bounded two-body potential. The existence proof for the "strong" solution of the evolution problem is obtained under suitable conditions on the initial state.
\end{abstract}

\section{Introduction}

In general, starting from a quasi-free (or generalized-free) state $\varrho$ of a finite or infinite Fermi system at the time $t=t_{0}$, the natural evolution of the system gives rise to a state $\varrho_{t}$ which does not remain quasi-free for $t>t_{0}$, and trustworthy methods of successive approximations for solving the evolution problem except in trivial cases are not known. An approximate procedure for solving this problem is provided by the time-dependent Hartree-Fock theory, first obtained by Dirac [1] and afterwards generalized by Bogoliubov [2] and Valatin [3]. These equations can be obtained by considering the evolution of the oneparticle density matrix $T$ and assuming that $\varrho_{t}$ remains quasi-free in a given time interval. Perturbative solutions of such equations for superconducting systems have been studied by Di Castro and Young [4].

In spite of the simplicity of the approach, the equation of motion for the one-particle density matrix $T$ is non-linear so that the existence problem is not easy even in the most simple physical cases. Written in 
matrix form the equation in the gauge-invariant case is of the type (see e.g. Ref. [5]):

$$
i \frac{d T}{d t}=[A+U, T]
$$

where $A$ is the kinetic energy operator and $U$ is the self-consistent potential which is a linear function of T. $U$ is the difference of two terms: $U=U_{D}-U_{E X}$, where $U_{D}$ denotes the "local" part and $U_{E X}$ the exchange part. Neglecting the spin coordinates, which are completely unessential for our purposes, and denoting by $q$ the space coordinate, by $\varphi$ a oneparticle wave-function, by $v\left(q, q^{\prime}\right)$ the two-body potential, and by $T\left(q, q^{\prime}\right)$ the "matrix element" of $T$ in the coordinate representation, we have:

$$
\begin{aligned}
\left(U_{D} \varphi\right)(q) & =\left[\int v\left(q, q^{\prime}\right) T\left(q^{\prime}, q^{\prime}\right) d^{3} q^{\prime}\right] \varphi(q) \\
\left(U_{E X} \varphi\right)(q) & =-\int v\left(q, q^{\prime}\right) T\left(q, q^{\prime}\right) \varphi\left(q^{\prime}\right) d^{3} q^{\prime} .
\end{aligned}
$$

Of course, Eq. (1.1) has to be solved with the given initial condition $\left.T\right|_{t=0}=T_{0}$.

We give here an existence and uniqueness proof for the solution of Eq. (1.1), assuming that the total number of particles is finite $\left(N=\int T(q, q) d^{3} q<+\infty\right)$ and the two-particle potential $v\left(q, q^{\prime}\right)$ is bounded: $\sup _{q, q^{\prime}}\left|v\left(q, q^{\prime}\right)\right|<+\infty$.

\section{Notations and Hypotheses}

We denote by:

$E$ a Hilbert space with inner product $\langle\cdot, \cdot\rangle$;

$\mathscr{L}(E)$ the set of all bounded linear operators defined in $E$, equipped with the norm topology $\|\cdot\|$.

$\mathscr{L}_{1}(E) \subset \mathscr{L}(E)$ the set of trace-class operators, equipped with the usual norm $\|\cdot\|_{1}=\operatorname{Tr}|\cdot|$.

$\mathscr{L}\left(\mathscr{L}_{1}(E), \mathscr{L}(E)\right)$ the Banach space of all linear continuous mappings $\mathscr{L}_{1}(E) \rightarrow \mathscr{L}(E)$, equipped with the usual norm $\| \cdot|| \mid$ topology.

$$
\begin{aligned}
H(E) & =\left\{T, T \in \mathscr{L}(E), T=T^{*}\right\} \\
H_{1}(E) & =\left\{T, T \in \mathscr{L}_{1}(E), T=T^{*}\right\} \\
C\left(0, \tau ; H_{1}(E)\right) & =\left\{f ; f:[0, \tau] \rightarrow H_{1}(E), f \text { continuous }\right\}
\end{aligned}
$$

where $\tau>0 ; C$ is a real Banach space equipped with the norm $\|f\|=\sup \left\{\|f(t)\|_{1}, t \in[0, \tau]\right\}$.

Let $\tau \in \mathbb{R}_{+}, \quad T_{0} \in H_{1}(E), \quad A: D_{A}(\cong E) \rightarrow E$ a self-adjoint operator, $B \in \mathscr{L}\left(\mathscr{L}_{1}(E), \mathscr{L}(E)\right)$ such that:

$$
T \in H_{1}(E) \rightarrow B(T) \in H(E) .
$$


We consider the following problem: find a function $T(\cdot) \in C\left(0, \tau ; H_{1}(E)\right)$ such that:

$$
\left\{\begin{aligned}
i \frac{d T}{d t} & =[A, T]_{-}+[B(T), T]_{-} \\
T(0) & =T_{0} .
\end{aligned}\right.
$$

Definition 2.1. A function $T \in C\left(0, \tau ; H_{1}(E)\right)$ is called a mild solution of the problem (2.2) if the following equality holds:

$$
T(t) x=e^{-i t A} T_{0} e^{i t A} x+i \int_{0}^{t} e^{-i(t-s) A}[T(s), B(T(s))]_{-} e^{i(t-s) A} x d s
$$

for every $x \in E$.

Definition 2.2. A function $T \in C\left(0, \tau ; H_{1}(E)\right)$ is called a classical solution of problem (2.2) if the following conditions are satisfied:

i) $T(\cdot)$ is continuously differentiable on the interval $[0, \tau]$;

ii) $\forall x \in D_{A}, \forall t \in[0, \tau]$, we have $T(t) x \in D_{A}$ and

$$
\left\{\begin{aligned}
i \frac{d T(t)}{d t} x & =A T(t) x-T(t) A x+[B(T(t)), T(t)]_{-} x \\
T(0) x & =T_{0} x .
\end{aligned}\right.
$$

It is easy to show that if $A$ is a bounded operator defined on $E$ the mild solution is also a classical solution.

\section{Preliminary Results}

Definition 3.1. For every $T \in H_{1}(E)$ we define a mapping $\varphi_{T}: D_{A}$ $\times D_{A} \rightarrow \mathbb{C}$ by the following relation:

$$
\varphi_{T}(x, y)=-i\langle T x, A y\rangle+i\langle A x, T y\rangle, \forall(x, y) \in D_{A} \times D_{A} .
$$

If $\varphi_{T}$ is continuous on $D_{A} \times D_{A}$ with respect to the product topology, we denote by the same symbol the unique extension to $E \times E$ of $\varphi_{T}$.

Definition 3.2. Let $a$ be the linear mapping defined by

$$
\left\{\begin{aligned}
D_{a}=\{T ; T \in & H_{1}(E), \varphi_{T} \text { is continuous with } \\
& \text { respect to the product topology of } E \times E\} \\
\langle a(T) x, y\rangle= & \varphi_{T}(x, y) \forall T \in D_{a}, \forall(x, y) \in E \times E .
\end{aligned}\right.
$$

It is easy to show that $T \in D_{a}, x \in D_{A}$ implies $T x \in D_{A}$ and the following equality holds

$$
a(T) x=-i A T x+i T A x
$$

(see Ref. [8]). 
Lemma 3.3. Let a have the same meaning as before; then the spectrum $\sigma(a) \subset i \mathbb{R}$ and

$$
\begin{gathered}
(\lambda-a)^{-1}(T) x=\int_{0}^{\infty} e^{-\lambda t} e^{-i t A} T e^{i t A} x d t, \\
\forall \lambda \in \mathbb{C}, \operatorname{Re} \lambda>0, \forall x \in E, T \in H_{1}(E) .
\end{gathered}
$$

Proof. A detailed proof of relation (3.4) can be found in Ref. [8]. The statement $\sigma(a) \subset i \mathbb{R}$ then follows easily.

Proposition 3.4. $a$ is the infinitesimal generator of a contraction semigroup in $H_{1}(E)$ and the following relation holds:

$$
e^{t a}(T)=e^{-i t A} T e^{i t A}, \quad \forall T \in H_{1}(E) .
$$

Proof. Since $e^{i t A}$ is unitary, we have

$$
\left\|e^{-i t A} T e^{i t A}\right\|_{1}=\|T\|_{1} \text {. }
$$

The semigroup property can be checked in a trivial way, so that we have only to prove that:

$$
\lim _{t \rightarrow 0^{+}} e^{-i t A} T e^{i t A}=T \quad \forall T \in H_{1}(E) .
$$

Since the set of finite rank operators is dense in $\mathscr{L}_{1}(E)$ in the tracenorm topology $\|\cdot\|_{1}$, we can restrict ourselves to prove Eq. (3.7) for an arbitrary projection operator of rank one.

Let $T$ be defined by

$$
T x=\langle x, y\rangle y \quad \forall x \in E,\|y\|=1 .
$$

We have:

$$
\left(e^{-i t A} T e^{i t A}-T\right) x=\left\langle x, e^{-i t A} y\right\rangle e^{-i t A} y-\langle x, y\rangle y .
$$

The two-dimensional subspace generated by $y$ and $e^{-i t A} y$ is invariant with respect to the operator $e^{-i t A} T e^{i t A}-T$; so the eigenvalue problem is easily solved and one finds for the non-vanishing eigenvalues of $e^{-i t A} T e^{i t A}-T$ :

$$
\lambda= \pm\left(1-\left|\left\langle e^{-i t A} y, y\right\rangle\right|^{2}\right)^{\frac{1}{2}} .
$$

It follows that

$$
\left\|e^{-i t A} T e^{i t A}-T\right\|_{1}=2 \sqrt{1-\left|\left\langle e^{-i t A} y, y\right\rangle\right|^{2}} \underset{t \rightarrow 0^{+}}{\longrightarrow} 0 .
$$

Hence the semigroup defined by (3.7) is strongly continuous. By Lemma $3.3 a$ is the infinitesimal generator of this semigroup.

Let

$$
\gamma(T)=-i[B(T), T] \quad \forall T \in H_{1}(E)
$$


then $\gamma: H_{1}(E) \rightarrow H_{1}(E)$ is a continuous mapping and

$$
\|\gamma(T)\|_{1} \leqq 2\|B\| \|\left(\|T\|_{1}\right)^{2} .
$$

Proposition 3.5. The following statements are true:

i) $\gamma$ is locally lipschitzian on $H_{1}(E)$.

ii) $\gamma$ is differentiable and

$$
\gamma^{\prime}(T) \cdot S=-i[B(S), T]-i[B(T), S] .
$$

iii) The following inequality holds

$$
\|T\|_{1} \leqq\|T-\alpha \gamma(T)\|_{1}, \quad \forall T \in H_{1}(E), \forall \alpha \in \mathbb{R}_{+} .
$$

Proof. i) Let $\|T\|_{1},\|S\|_{1} \leqq r, r>0$; then

$$
\begin{aligned}
\|\gamma(T)-\gamma(S)\|_{1} & =\left\|[B(T), T]_{-}-[B(T), S]_{-}+[B(T), S]_{-}-[B(S), S]_{-}\right\|_{1} \\
& \leqq\left\|[B(T), T-S]_{-}\right\|_{1}+\left\|[B(T-S), S]_{-}\right\|_{1} \\
& \leqq 4\|B\| r\|T-S\|_{1} ;
\end{aligned}
$$

ii) can be directly verified.

iii) Let $\alpha>0, T \in H_{1}(E)$, and

$$
T-\alpha \gamma(T)=S \text {. }
$$

Denoting by $\left\{\lambda_{i}\right\}$ the set of the eigenvalues of $T$ and by $\left\{u_{i}\right\}$ a corresponding set of orthonormal eigenvectors, we can write:

$$
T x=\sum_{i=1}^{\infty} \lambda_{i}\left\langle x, u_{i}\right\rangle u_{i} .
$$

Defining:

$$
\begin{gathered}
\sigma(T) x=\sum_{i=1}^{\infty} \operatorname{sign}\left(\lambda_{i}\right)\left\langle x, u_{i}\right\rangle u_{i} \\
|T| x=\sum_{i=1}^{\infty}\left|\lambda_{i}\right|\left\langle x, u_{i}\right\rangle u_{i}
\end{gathered}
$$

since

$$
\operatorname{Tr}[\gamma(T) \sigma(T)]=\operatorname{Tr}[\sigma(T) \gamma(T)]=0
$$

it follows that:

$$
\begin{gathered}
\|T\|_{1}=\frac{1}{2} \operatorname{Tr}(S \sigma(T)+\sigma(T) S) \\
\leqq \frac{1}{2} \operatorname{Tr}(|S \sigma(T)+\sigma(T) S|) \leqq\|\sigma(T)\|\|S\|_{1}=\|S\|_{1}
\end{gathered}
$$

which proves (3.10). 


\section{The Existence Theorem}

Let $X$ be a real Banach space (with norm \|\|$\left._{X}\right), C(0, \tau ; X)$ the Banach space of the continuous mappings $[0, \tau] \rightarrow X$ equipped with the norm $\|\cdot\|=\operatorname{Sup}\left\{\|\cdot(t)\|_{X}, t \in[0, \tau]\right\}, M$ the infinitesimal generator of a contraction semigroup $t \rightarrow e^{t M}$ in $X . f: X \rightarrow X$ a locally lipschitzian mapping ${ }^{1}$ such that:

$$
\|x\|_{X} \leqq\|x-\alpha f(x)\|_{X} \quad \forall \alpha \geqq 0, x \in X .
$$

We consider the following integral equation:

$$
u(t)=e^{t M} u_{0}+\int_{0}^{t} e^{(t-s) M} f[u(s)] d s
$$

where $u_{0}$ is a given element in $X$ and $u \in C(0, \tau ; X)$.

Then the following theorem holds: (for the proof see Refs. $[6,7,11]$ ).

Theorem 4.1. There exists a unique solution of the problem (4.2). This solution depends continuously upon the initial condition. Furthermore, if $u_{0} \in D_{M}$ and is differentiable in $X$, then $u$ is differentiable in $[0, \tau]$, $u(t) \in D_{M} \forall t \in[0, \tau]$ and we have

$$
\left\{\begin{aligned}
\frac{d u(t)}{d t} & =M u(t)+f[u(t)] \\
u(0) & =u_{0} .
\end{aligned}\right.
$$

Applying Theorem 4.1 to our case, we obtain:

Theorem 4.2. $\forall T_{0} \in H_{1}(E)$ there exists a unique mild solution $T(\cdot)$ of Eq. (2.2). Furthermore, if the mapping

$$
(x, y) \rightarrow\left\langle T_{0} x, A y\right\rangle+\left\langle A x, T_{0} y\right\rangle \quad \forall(x, y) \in D_{A} \times D_{A}
$$

is continuous with respect to the product topology of $E \times E$, then $T(\cdot)$ is a classical solution which depends continuously upon the initial condition.

Proof. It is enough to apply Theorem 4.1 with $f=\gamma, M=a, X=H_{1}(E)$ and use Propositions 3.4, 3.5.

Proposition 4.3. If $T(\cdot)$ is a mild solution of problem (2.2) then for any $t \in[0, \tau]$ there exists a self-adjoint operator $K(t)$ such that

$$
T(t)=e^{-i K(t)} T_{0} e^{i K(t)} .
$$

Proof. Let $T_{0} \in D_{a}$ and $T(\cdot)$ be the classical solution of problem (2.2). We put $Q(t)=B(T(t)), t \in[0, \tau] ; Q$ is a Lipschitz continuous mapping $[0, \tau] \rightarrow H(E)$. It is easy to see that for the linear problem

$$
\left\{\begin{array}{l}
i \frac{d u}{d t}=(A+B(T(t))) u(t) \\
u\left(t_{0}\right)=u_{0}
\end{array}\right.
$$

${ }^{1}$ By locally lipschitzian we mean that for any $r>0, u \in X, v \in X,\|u\|_{X} \leqq r,\|v\|_{X} \leqq r$, $\exists N_{r}>0$ such that $\|f(u)-f(v)\|_{X} \leqq N_{r}\|u-v\|_{X}$. 
there exists a unitary Green function $U(t, s)$. It follows [8] that the problem

$$
\left\{\begin{aligned}
i \frac{d S(t)}{d t} & =[A+B(T(t)), S(t)]_{-} \\
S(0) & =T_{0}
\end{aligned}\right.
$$

has a unique classical solution given by

$$
S(t)=U(t, 0) T_{0} U(-t, 0) .
$$

Furthermore $T(\cdot)$ is obviously a solution of (4.6), so that, from the uniqueness of the solution, we have $S=T$.

For any $t \in[0, \tau]$ let $K(t)$ be the self-adjoint operator such that $U(-t, 0)=e^{i K(t)}$; Eq. (4.4) then follows.

If $T_{0} \in H_{1}(E)$ we can prove (4.7) by a straightforward argument of density, since $D_{a}$ is dense in $H_{1}(E)$.

\section{The Hartree-Fock Time-dependent Problem}

We now give sufficient conditions in order that Eq. (1.1) be solvable by the methods of Section 4.

Let $E=\mathscr{L}^{2}\left(R^{3}\right)$ be the one-particle Hilbert space. We assume that the two-particle potential $v\left(q, q^{\prime}\right)$

$$
v: \mathbb{R}^{3} \times \mathbb{R}^{3} \rightarrow \mathbb{R}
$$

is a real bounded measurable function verifying the conditions:

$$
\begin{aligned}
v\left(q, q^{\prime}\right) & =v\left(q^{\prime}, q\right) \\
\left|v\left(q, q^{\prime}\right)\right| & \leqq V, \forall q, q^{\prime} \in \mathbb{R}^{3} .
\end{aligned}
$$

Let $\left\{\varphi_{k}\right\}$ be a complete orthonormal system in $E$. We write the oneparticle density matrix in the form

$$
T\left(q, q^{\prime}\right)=\sum_{k=1}^{\infty} \lambda_{k} \varphi_{k}(q) \overline{\varphi_{k}\left(q^{\prime}\right)}
$$

The positivity condition for the gauge-invariant quasi-free state defined by $T$ implies $[9,10]$

$$
0 \leqq \lambda_{k} \leqq 1
$$

Since we consider only systems with finite total number of particles, we have

$$
\sum_{k=1}^{\infty} \lambda_{k}<\infty .
$$


$T\left(q, q^{\prime}\right)$ determines an operator $T \in H_{1}(E)$ such that

$$
T \psi=\sum_{k=1}^{\infty} \lambda_{k}\left(\psi, \varphi_{k}\right) \varphi_{k}
$$

Of course

$$
\|T\|_{1}=\sum_{k=1}^{\infty} \lambda_{k}=\int_{\mathbb{R}^{3}} T(q, q) d^{3} q .
$$

We define

$$
B_{D}(\cdot): H_{1}(E) \rightarrow H(E), \quad B_{E X}(\cdot): H_{1}(E) \rightarrow H(E)
$$

by the equalities

$$
B_{D}(T) \varphi=U_{D} \varphi, B_{E X}(T) \varphi=U_{E X} \varphi \quad \forall \varphi \in E
$$

where $U_{D}$ and $U_{E X}$ are given by (1.2), (1.3) respectively.

It is easy to see that $B_{D}$ is bounded and

$$
\left\|B_{D}\right\| \leqq V .
$$

Since

$$
\begin{aligned}
\left\|B_{E X}(T)\right\| & \leqq\left(\int_{\mathbb{R}^{3} \times \mathbb{R}^{3}}\left|v\left(q, q^{\prime}\right) T\left(q, q^{\prime}\right)\right|^{2} d^{3} q d^{3} q^{\prime}\right)^{\frac{1}{2}} \\
& \leqq V\left(\sum_{k=1}^{\infty} \lambda_{k}^{2}\right)^{\frac{1}{2}} \leqq V\|T\|_{1}
\end{aligned}
$$

also $\left\|B_{E X}\right\| \leqq V$, so that $B(T)=B_{D}(T)+B_{E X}(T)$ satisfies the hypotheses of Section 2. Hence the existence theorem applies and Proposition 4.3 guarantees that $T(t), t \in] 0, \tau]$ satisfies the positivity condition (5.4) if $T_{0}$ satisfies (5.4). Hence $T(t)$ defines a quasi-free state. Furthermore the state remains pure if it is initially pure $\left(T_{0}^{2}=T_{0}\right)$.

The existence of the strong solution is guaranteed by the following condition on the initial state

$$
R_{T} \subseteq D_{A} .
$$

This condition is physically reasonable in the greatest majority of the applications, where $A$ is either the kinetic energy operator, or the kinetic energy plus a central field. If (5.11) holds, $A T_{0}$ is bounded so that Eq. (3.3) holds.

Acknowledgements. The authors are very much indebted to Professors S. Doplicher, G. Dell'Antonio, and to Professor N. M. Hugenholtz for helpful discussions and criticism. 


\title{
References
}

1. Dirac, P.A. M.: Proc. Cambridge Phil. Soc. 26, 376 (1930)

2. Bogoliubov, N. N.: Uspekhi Fiz. Nauk. 67, 549 (1959)

3. Valatin, J.G.: Phys. Rev. 122, 1012 (1961); Lectures in theoretical physics, Vol. 6, p. 292. Boulder: W. E. Brittin 1963

4. Di Castro, C., Young, W.: Nuovo Cimento 62 B, 273 (1969)

5. Thouless, D.J.: The quantum mechanics of many-body systems, second edition. New York, London: Acad. Press 1972

6. Da Prato, G.: J. Math. Pures Appl. 49, 289 (1970)

7. Da Prato, G.: Istituto Nazionale di Alta Matematica, Symposia Mathematica 7, 233 (1971)

8. Da Prato, G.: Quelques résultats d'existence, unicité et régularité pour un problème de la théorie du contrôle, J. Math. Pures Appl., to appear (1973)

9. Fano, G.: Séminaires de l'Institut de Physique Théorique, C.N.R.S., Chemin J. Aiguier, Marseille (France), p. 194 III/1968

10. Powers, R.T.: Representations of the C.A.R. Princeton Thesis, 1967

11. Iannelli, M.: Boll. U.M.I. 6, 1015 (1970)

Communicated by W. Hunziker

\author{
A. Bove \\ Istituto di Fisica \\ dell' Università \\ di Bologna \\ Via Irnerio 46 \\ I-40126 Bologna, Italy
}


